\title{
Activation of the Notch-1 signaling pathway may be involved in intracerebral hemorrhage-induced reactive astrogliosis in rats
}

\author{
Jian-Hua Zhong, MD, ${ }^{1}$ Hua-Jun Zhou, MD, PhD, ${ }^{2,3}$ Tao Tang, MD, PhD, ${ }^{4}$ Han-Jin Cui, MD, PhD, ${ }^{4}$ \\ A-Li Yang, MD, PhD, ${ }^{5}$ Qi-Mei Zhang, MD, ${ }^{2,3}$ Jing-Hua Zhou, MD, PhD, ${ }^{2,3}$ Qiang Zhang, MD, PhD, ${ }^{2,3}$ \\ Xun Gong, MD, ${ }^{1}$ Zhao-Hui Zhang, MD, PhD, ${ }^{1}$ and Zhi-Gang Mei, MD, PhD ${ }^{6}$

\begin{abstract}
${ }^{1}$ Intensive Care Unit and ${ }^{3}$ Department of Neurology, The First College of Clinical Medical Sciences; ${ }^{2}$ nstitute of Neurology, and ${ }^{6}$ Medical College, China Three Gorges University, Yichang, Hubei; and ${ }^{4}$ nstitute of Integrative Medicine and ${ }^{5}$ Department of
\end{abstract} \\ Hyperbaric Oxygen, Xiangya Hospital, Central South University, Changsha, Hunan, People's Republic of China
}

\begin{abstract}
OBJECTIVE Reactive astrogliosis, a key feature that is characterized by glial proliferation, has been observed in rat brains after intracerebral hemorrhage $(\mathrm{ICH})$. However, the mechanisms that control reactive astrogliosis formation remain unknown. Notch-1 signaling plays a critical role in modulating reactive astrogliosis. The purpose of this paper was to establish whether Notch-1 signaling is involved in reactive astrogliosis after ICH.

METHODS ICH was induced in adult male Sprague-Dawley rats via stereotactic injection of autologous blood into the right globus pallidus. $N$-[N-(3,5-difluorophenacetyl)-L-alanyl]-S-phenylglycine $t$-butyl ester (DAPT) was injected into the lateral ventricle to block Notch-1 signaling. The rats' brains were perfused to identify proliferating cell nuclear antigen (PCNA)-positive/GFAP-positive nuclei. The expression of GFAP, Notch-1, and the activated form of Notch-1 (Notch intracellular domain [NICD]) and its ligand Jagged-1 was assessed using immunohistochemical and Western blot analyses, respectively.
\end{abstract}

RESULTS Notch-1 signaling was upregulated and activated after ICH as confirmed by an increase in the expression of Notch-1 and NICD and its ligand Jagged-1. Remarkably, blockade of Notch-1 signaling with the specific inhibitor DAPT suppressed astrocytic proliferation and GFAP levels caused by $\mathrm{ICH}$. In addition, DAPT improved neurological outcome after $\mathrm{ICH}$.

CONCLUSIONS Notch-1 signaling is a critical regulator of $\mathrm{ICH}$-induced reactive astrogliosis, and its blockage may be a potential therapeutic strategy for hemorrhagic injury.

https://thejns.org/doi/abs/10.3171/2016.11.JNS162121

KEY WORDS intracerebral hemorrhage; reactive astrogliosis; Notch-1 signaling; glial scar

I NTRACEREBRAL hemorrhage (ICH), accounting for at least $10 \%$ of all strokes in the Western population ${ }^{6}$ and a considerably higher proportion in the Asian and black populations, ${ }^{22}$ results in severe neurological damage in survivors with no effective treatment. Therefore, a better understanding of the pathophysiology of $\mathrm{ICH}$ might lead to better clinical management of patients with $\mathrm{ICH}$.

Astrocytes are prominent brain cells and provide a variety of critical supportive functions that maintain neuronal homeostasis. However, after CNS injury, astrocytes respond to all forms of insults by a process commonly referred to as reactive astrogliosis characterized by cellular hypertrophy, enhanced astrocyte proliferation, and upregulation of intermediate filament proteins, such as GFAP. ${ }^{18}$ In severe cases, reactive astrogliosis eventually leads to the formation of a glial scar surrounding the injury site. Al-

ABBREVIATIONS AUR = asymmetrical use rate; DAPT = N-[N-(3,5-difluorophenacetyl)-L-alanyl]-S-phenylglycine $t$-butyl ester; $I C H=$ intracerebral hemorrhage; NICD = Notch intracellular domain; PCNA = proliferating cell nuclear antigen.

SUBMITTED August 15, 2016. ACCEPTED November 21, 2016.

INCLUDE WHEN CITING Published online October 27, 2017; DOI: 10.3171/2016.11.JNS162121. 
though a glial scar may isolate and protect the noninjured tissue from exposure to the toxic elements, inhibit the spread of inflammation, and regulate the extracellular milieu, it may also create a physical barrier and a biochemical barrier to axonal regeneration, which, in turn, hinders the functional recovery process. ${ }^{16}$ Therefore, modulation of reactive astrogliosis and glial scar is an important potential therapeutic strategy after brain injuries. A previous study demonstrated that reactive astrogliosis occurred after ICH. ${ }^{20}$ However, the signaling pathways that control reactive astrogliosis after ICH are poorly defined.

Notch receptors and their ligands, molecules best known for influencing cell fate decisions through direct cell-cell contact, ${ }^{1}$ play a critical role in endowing neural precursor cells with the ability to differentiate into astrocytes. ${ }^{13}$ It has been reported that Notch-1 signaling activation leads to increased astrogliosis in vitro ${ }^{28}$ and in vivo. ${ }^{4}$ Furthermore, a body of evidence indicated that Notch-1 or its ligand Jagged-1 positively regulated astrocyte proliferation and enhanced reactive astrogliosis after CNS injury, including cerebral ischemia, brain trauma, and spinal cord injury, ${ }^{2,7,9,17}$ However, the effect of Notch-1 signaling on reactive astrogliosis after ICH is not known. Accordingly, the purpose of this paper was to establish whether Notch-1 signaling is involved in reactive astrogliosis following $\mathrm{ICH}$.

\section{Methods \\ Animal Preparation}

Adult male Sprague-Dawley rats $(n=120)$ weighing 250-300 g were housed under diurnal lighting conditions. This study was carried out in strict accordance with the recommendations in the Guide for the Care and Use of Laboratory Animals of the National Institutes of Health (NIH Publication No. 85-23, revised 1996). All experiments were approved by the Institutional Animal Care and Use Committee of China Three Gorges University.

\section{Surgical Procedure of $\mathrm{ICH}$}

ICH was induced as described in detail previously. ${ }^{27}$ Animals were anesthetized with an intraperitoneal injection of chloral hydrate $(400 \mathrm{mg} / \mathrm{kg})$ and then fixed prone onto a stereotactic frame (Stoelting Co.). After a scalp incision was made, a small cranial bur hole was drilled near the right coronal suture $3.2 \mathrm{~mm}$ lateral to the midline. A 26-gauge needle was inserted stereotactically into the right globus pallidus ( $1.4 \mathrm{~mm}$ posterior and $3.2 \mathrm{~mm}$ lateral to the bregma, $5.6 \mathrm{~mm}$ ventral to the cortical surface). One hundred microliters of nonheparinized autologous whole blood was taken from the femoral artery and injected into the globus pallidus over 2 minutes, and the needle was slowly removed over another 10 minutes to prevent backflow.

\section{Experimental Designs}

This study was divided into 2 parts. In part 1, 60 rats were randomly assigned to the sham group or the ICH group. In the ICH group, the rats received $100 \mu \mathrm{l}$ autologous blood (ICH) into the right globus pallidus. Rats in the sham group underwent needle insertion only. In part 2, the goal was to further define the role of Notch-1 signaling in ICH-induced reactive astrogliosis in vivo. The gamma-secretase inhibitor $N-[N-(3$, 5 -difluorophenacetyl)-L-alanyl]- $S$-phenylglycine $t$-butyl ester (DAPT; Sigma-Aldrich) was used to block Notch-1 signaling. DAPT powder was dissolved in $0.01 \mathrm{M}$ phosphate-buffered saline including 5\% dimethyl sulfoxide (Sigma-Aldrich Inc.) to prepare concentrations of $8.3 \mathrm{mg} /$ $\mathrm{ml}$. All rats in part $2(\mathrm{n}=60)$ had undergone induction of ICH. One group of rats received DAPT $(0.03 \mathrm{mg} / \mathrm{kg})$ via direct infusion into the right lateral cerebral ventricle (0.8 $\mathrm{mm}$ posterior, $-4.8 \mathrm{~mm}$ dorsoventral, and $-1.5 \mathrm{~mm}$ lateral to the bregma) immediately after ICH. ${ }^{10}$ The control group received an equal volume of vehicle. The rats' brains were perfused to identify proliferating cell nuclear antigen (PCNA)-positive/GFAP-positive nuclei and the expression of GFAP, Notch-1, and the activated form of Notch-1 (Notch intracellular domain [NICD]) and its ligand Jagged-1 was evaluated using immunohistochemical and Western blot analyses, respectively.

\section{Specimen Preparation}

The rats were deeply anesthetized with chloral hydrate $(800 \mathrm{mg} / \mathrm{kg})$. For immunohistochemical analysis, the rats ( $\mathrm{n}=5$ per time point $[3,7$, and 14 days $]$ ) were transcardially perfused with $0.9 \%$ saline followed by $250 \mathrm{ml}$ icecold $4 \%$ paraformaldehyde in $0.1-\mathrm{M}$ phosphate buffer $(\mathrm{pH}$ 7.4). Rats in the sham group were killed on Day 3. The brains were excised and postfixed in the same fixative for 2 hours and then embedded in paraffin. All tissues were cut into 5- $\mu \mathrm{m}$ coronal sections. For Western blot analysis, after perfusion ( $\mathrm{n}=5$ per time point), the brains were immediately removed, and the tissues in the striatum adjacent to the hematoma were dissected and stored at $-196^{\circ} \mathrm{C}$ in liquid nitrogen.

\section{Neurological Evaluation}

Neurological deficits induced by ICH were assessed using a forelimb asymmetry test. Briefly, rats were videotaped in a $20-\mathrm{cm}$-diameter and $30-\mathrm{cm}$-tall transparent cylinder. A mirror was placed on the side of the cylinder at an angle convenient for observing the forelimb movement of the rat; at the same time, recording was performed using a video camera. The test continued for 10 minutes. In the testing time, behavior was quantified by counting the occasions that the rat's forelimb touched the cylinder wall while it was in an orthostatic position with a balanced gravity center. The occasions of contact with the unimpaired (ipsilateral) forelimb and impaired forelimb (contralateral to the autologous blood injection site) were recorded as I and C, respectively. The occasions of contact with both forelimbs simultaneously were recorded as B. The forelimb asymmetrical use rate (AUR) was calculated as follows: $\mathrm{AUR}=[\mathrm{I} /(\mathrm{I}+\mathrm{C}+\mathrm{B})]-[\mathrm{C} /(\mathrm{I}+\mathrm{C}+\mathrm{B})] .^{5}$

\section{Immunohistochemical Analysis \\ Evaluation of Reactive Astrogliosis}

The expression of GFAP was detected by immunofluorescence as previously described. ${ }^{3}$ The primary antibody was mouse anti-GFAP (1:500, Santa Cruz Biotech). 
AF488-conjugated goat anti-mouse antibody (1:100, Jackson ImmunoResearch Laboratories) was used as a secondary antibody. DAPI (10 mg/ml, Sigma-Aldrich Inc.) was included in the secondary antibody incubation for labeling nuclei. Finally, the slides were washed and coverslipped with antifade mounting media. The sections were observed under an Olympus BX51 fluorescent microscope connected to a computer screen.

To detect proliferated GFAP, double immunolabeling was performed. Cell proliferation was determined based on immunostaining of the endogenous cell proliferative marker PCNA, and astrocytes were determined based on the immunoreactivity of GFAP. The sections were first incubated for 1 hour at room temperature with a mixture of 2 primary antibodies against mouse anti-GFAP (1:250) and rabbit anti-PCNA (1:250, Santa Cruz Biotech). AF488-conjugated goat anti-mouse antibody (1:100) and Cy3-conjugated goat anti-rabbit antibody (1:100, Jackson ImmunoResearch Laboratories) were used as secondary antibodies. DAPI was used to label nuclei. The sections were scanned using a laser scanning confocal microscope (LSM-510, Zeiss). For a negative control, $1 \%$ bovine serum albumin was used instead of the primary antibody in each experiment.

GFAP-positive astrocytes and PCNA-positive/GFAPpositive nuclei close to the hematoma were counted in four $250 \times 250-\mu \mathrm{m}$ areas in 10 sections through the stroke region for each animal at $\times 40$ objective magnification using image analysis software (version 3.2, Motic Images Advance) by a researcher blinded to the experimental cohort, and the data were presented as the number of nuclei per $\mathrm{mm}^{2}$.

\section{Expression of Notch-1, NICD, and Jagged-1}

To observe spatial profiles of Notch-1, NICD, and Jagged-1, immunohistochemical analysis was used for detection of their expression. ${ }^{29}$ The primary antibodies were used as follows: goat polyclonal anti-Notch-1 (1:100, Santa Cruz Biotech), rabbit polyclonal anti-NICD (1:500, Abcam), and goat polyclonal anti-Jagged-1 (1:100, Santa Cruz Biotech).

To determine whether Notch-1, NICD, and Jagged-1 were coexpressed with GFAP, double immunolabeling was used. The sections were first incubated for 1 hour at room temperature with a mixture of 2 primary antibodies against mouse anti-GFAP (1:250) and one of the following antibodies: goat polyclonal anti-Notch-1 (1:50), rabbit polyclonal anti-NICD (1:250), and goat polyclonal anti-Jagged-1 (1:50). AF488-conjugated goat anti-mouse antibody (1:100) and Cy3-conjugated goat anti-rabbit and donkey anti-goat antibodies (1:100) were used as secondary antibodies. DAPI $(10 \mathrm{mg} / \mathrm{ml})$ was used to label nuclei. These sections were scanned using a laser scanning confocal microscope (LSM-510, Zeiss). For a negative control, $1 \%$ bovine serum albumin was used instead of the primary antibody in each experiment.

\section{Western Blot}

To detect the protein levels of GFAP, Notch-1, NICD, and Jagged-1, protein was extracted from the tissues in the striatum adjacent to the hematoma, and Western blot analysis was performed. ${ }^{21}$ Briefly, 50- $\mu \mathrm{g}$ proteins were separated using sodium dodecyl sulfate polyacrylamide gel electrophoresis and transferred to a Hybond-C pure nitrocellulose membrane (Amersham). Monoclonal mouse antibodies for GFAP (1:1000), polyclonal rabbit antibodies for NICD (1:500), and polyclonal goat antibodies for Notch-1, Jagged-1, and $\beta$-actin (1:200) were used. Quantitative densitometric analysis of Western blots was performed using a computerized digital image analysis system.

\section{Statistical Analysis}

All data in this study are presented as the mean \pm SD. Data were analyzed using the Student t-test and 1-way ANOVA, followed by Scheffe's post hoc test. Differences were considered significant at $\mathrm{p}<0.05$.

\section{Results}

\section{Reactive Astrogliosis After ICH}

Because increased GFAP immunoreactivity has been used extensively as a diagnostic indicator of CNS reactive astrogliosis, we performed immunostaining for GFAP to evaluate the astrocytic reaction. Resting (nonreactive) astrocytes with small cell bodies and fine terminal processes were observed in the sham group (Fig. 1A1). After ICH, several astrocytes became activated, with reactive, hypertrophied morphology on Day 3 (Fig. 1A2) and extensive overlap on Day 14 (Fig. 1A3). Furthermore, the number of GFAP-positive astrocytes increased sharply and reached the maximum level on Day 14 post-ICH (Fig. 1A4, p < $0.05)$.

It is generally accepted that reactive astrogliosis is characterized by increased cellular proliferation. Thus, we next determined whether the increase in GFAP immunoreactivity was associated with increased cellular proliferation after ICH. The cell proliferation was determined based on the immunostaining with the endogenous cell proliferative marker PCNA. In the sham group, PCNA-positive nuclei were sporadically observed, and few PCNA-positive/ GFAP-positive nuclei could be detected (Fig. 1B1). After ICH, quantitative analysis of PCNA-positive/GFAP-positive nuclei revealed an increase from Day 3 until Day 14 (Fig. 1B2 and B3, p < 0.01).

Finally, Western blotting was used to evaluate the expression of GFAP at the protein level. GFAP was expressed at low levels in sham rats. In contrast, a significant upregulation of GFAP was noted at Day 3, and the levels continued to increase until Day 14 post-ICH (Fig. 1C, p < $0.01)$.

\section{Notch-1 Signaling Is Activated After ICH}

To assess whether Notch-1 signaling is activated after $\mathrm{ICH}$, we initially performed immunohistochemical and Western blot analyses using anti-Notch-1 antibody, antiNICD antibody, and anti-Jagged-1 antibody. Compared with the sham group, the number of Notch-1-, NICD-, and Jagged-1-positive cells were notably elevated on Day 3 and peaked on Day 14 post-ICH. Double immunolabeling showed that Notch-1, NICD, and Jagged-1 immunoreactivity were colocalized with the astroglial marker GFAP (Fig. 2, p < 0.01). In line with results of immunohisto- 
A
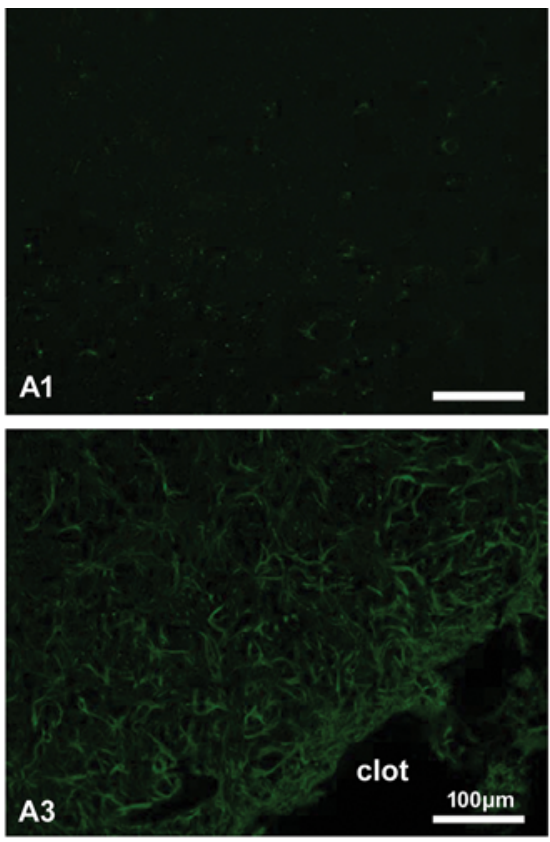

B

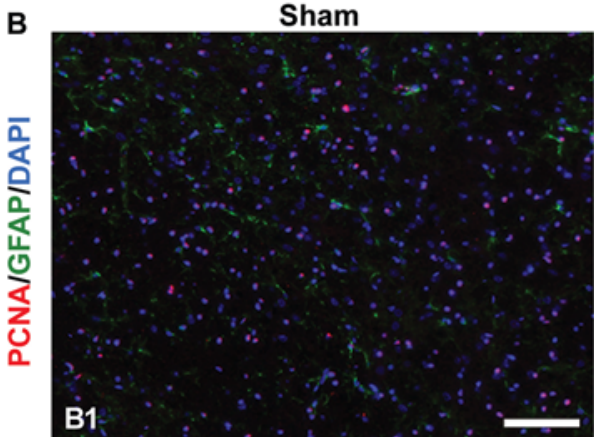

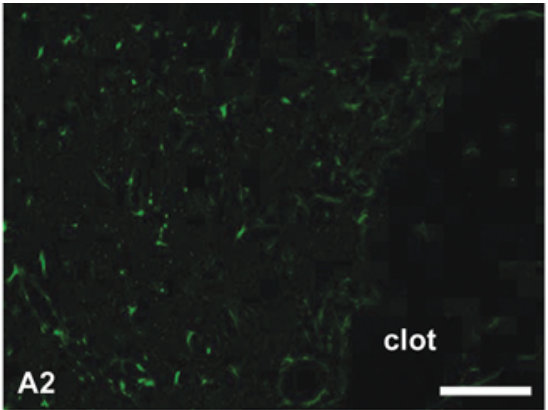

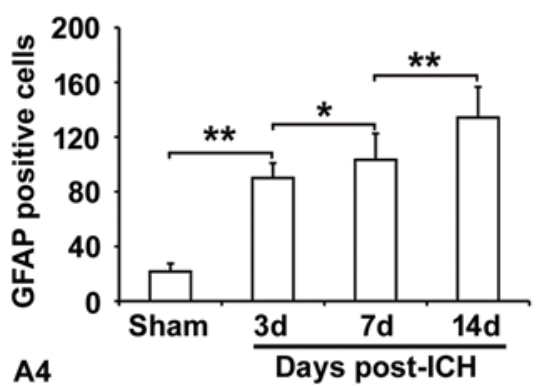

$\mathrm{ICH}$
C

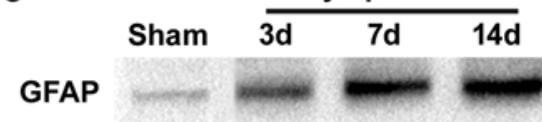

$\beta$-actin

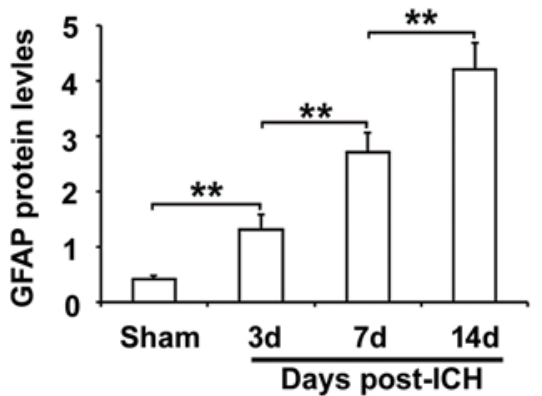

FIG. 1. Reactive astrogliosis after ICH. A: Resting astrocytes with small cell bodies and fine terminal processes are seen in the sham control group (A1). After ICH, several astrocytes became activated with reactive, hypertrophied morphology on Day 3 (A2) and extensive overlap on Day 14 (A3). Quantitative analysis showed that GFAP-positive astrocytes increased sharply and reached their maximum level on Day 14 post-ICH (A4). B: Immunofluorescent double labeling showing that few PCNA-positive/GFAPpositive nuclei are detected in the sham control group (B1). However, many PCNA-positive nuclei in GFAP' astrocytes can be seen around the hematoma (B2). Labeled nuclei in vessels increased until 14 days after ICH (B3). C: Similarly, Western blot analysis demonstrates that the upregulation of GFAP persisted until 14 days after ICH. ${ }^{*} p<0.05,{ }^{* *} p<0.01 ; n=5$ rats per time point. Scale bar $=100 \mu \mathrm{m}$.

chemistry, Western blotting showed a significant increase in Notch-1, NICD, and Jagged-1 proteins from Day 3 to Day 14 after ICH compared with the sham group (Fig. 3, $\mathrm{p}<0.01)$.

\section{Notch-1 Signaling Is Involved in ICH-Induced Reactive Astrogliosis}

To further determine whether Notch-1 signaling was responsible for ICH-induced reactive astrogliosis, DAPT, a $\gamma$-secretase inhibitor, was used to block Notch-1 signaling by injection into the right lateral ventricle after ICH. After DAPT treatment, not only was NICD expression downregulated (Fig. 4A, p < 0.05), but also the number of PCNA-positive/GFAP-positive nuclei decreased and GFAP protein levels were attenuated (Fig. 4B, $\mathrm{p}<0.05$ ). This suggested that activated Notch-1 signaling was involved in ICH-induced reactive astrogliosis.

\section{Inhibition of Notch-1 Signaling Ameliorated Neurological Deficits}

As described above, reactive astrogliosis could exert a beneficial or detrimental effect after CNS injury. ${ }^{16}$ Hence, we also evaluated the effect of Notch-1 signaling on neurological deficits after ICH. No obvious behavioral changes were found in the sham group. However, all ICH-group rats displayed similar and marked neurological impairments compared with the sham group at the corresponding time points. Reduction of AUR was significant in the DAPT-treated group compared with the control group (Fig. 5, p < 0.01).

\section{Discussion}

To our knowledge, findings provide the first evidence that activation of Notch-1 signaling contributes to ICH-induced 

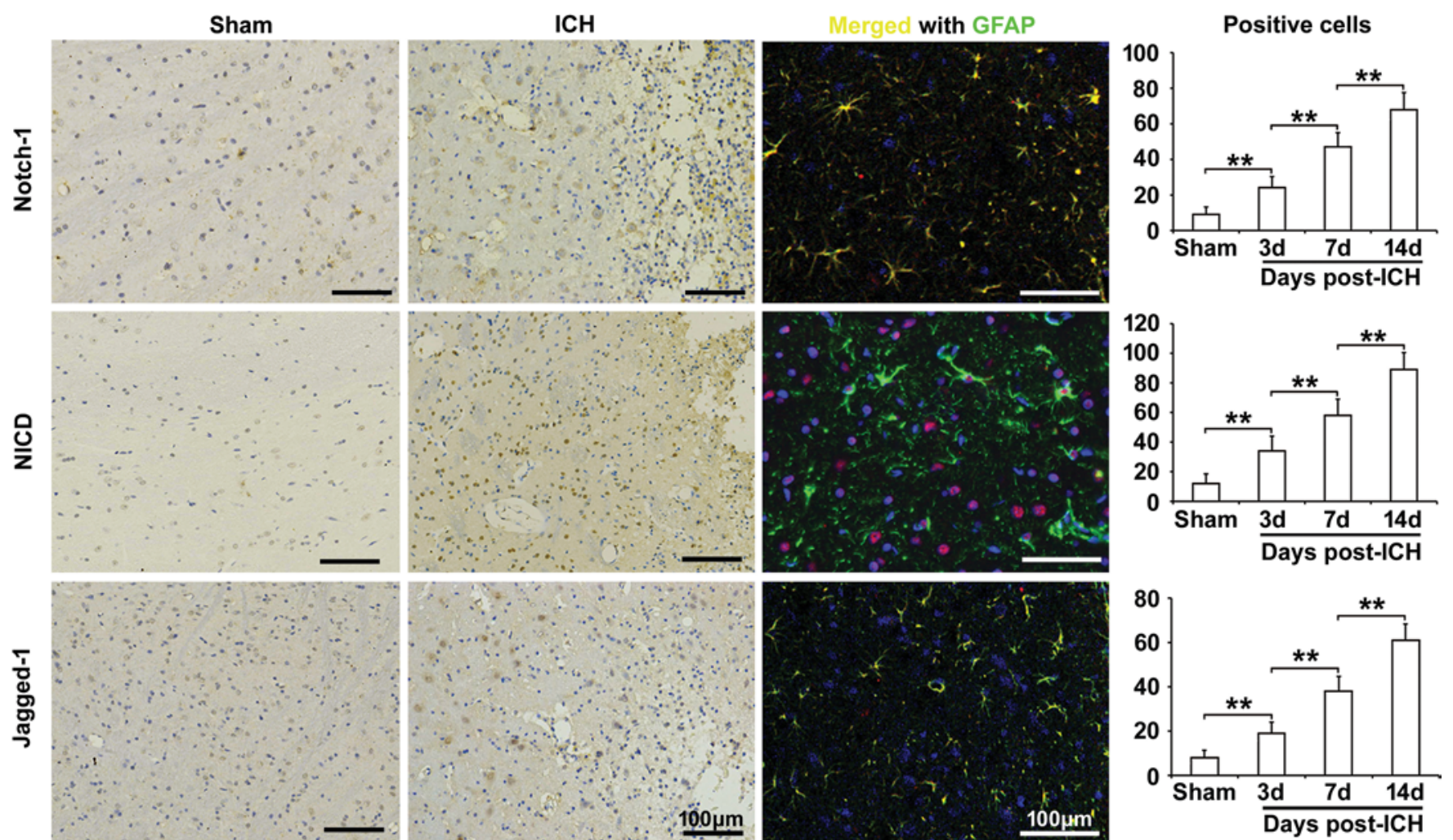

FIG. 2. Expression of Notch-1, NICD, and Jagged-1 after ICH. Immunohistochemical analysis of brain sections from sham or ICH mice was performed for Notch-1, NICD, and Jagged-1. Immunofluorescent double labeling showed that Notch-1, NICD, and Jagged-1 were localized in GFAP-positive astrocytes after ICH. Quantitative analysis showed that compared with the sham-control group the numbers of Notch-1-, NICD-, and Jagged-1-positive cells were notably elevated on Day 3 and peaked on Day 14 postICH. ${ }^{* *} p<0.01 ; n=5$ rats per time point. Scale bar $=100 \mu \mathrm{m}$.
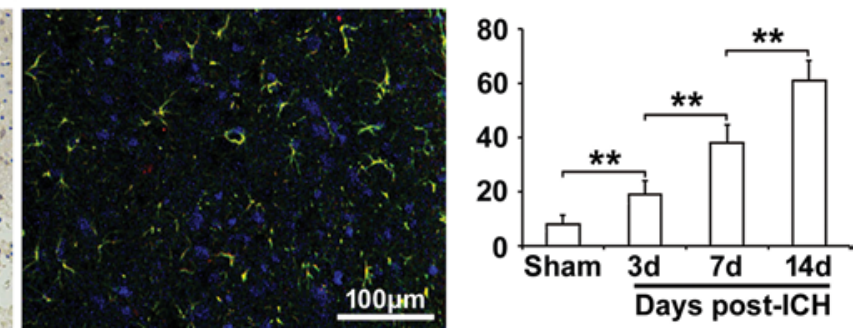

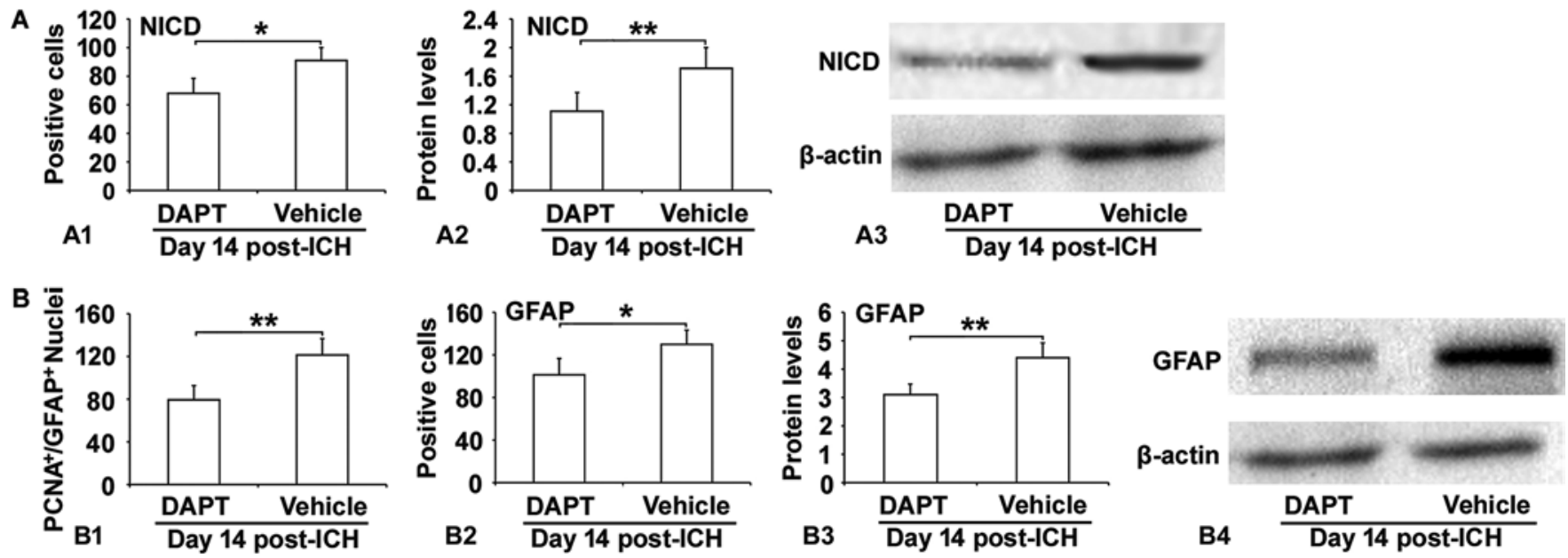

FIG. 4. Notch-1 signaling involved in ICH-induced reactive astrogliosis. A: After DAPT treatment, NICD-positive cells (A1) decreased and protein levels (A2 and A3) were reduced. B: DAPT decreased the number of PCNA-positive/GFAP-positive nuclei (B1) and GFAP-positive cells (B2) as well as downregulated GFAP protein levels (B3 and B4). ${ }^{*} p<0.05,{ }^{* *} p<0.01 ; n=5$ rats per time point.

reactive astrogliosis in rats, because increased PCNA-positive/GFAP-positive nuclei and GFAP protein levels were attenuated by DAPT, an inhibitor of Notch-1 signaling.

Notch-1 is a transmembrane receptor protein. Both Notch-1 and its cognate ligand Jagged-1 are expressed in the adult mammalian brain. ${ }^{19}$ Upon ligand binding, Notch-1 is cleaved by the $\gamma$-secretase complex and releases an NICD that translocates into the nucleus and activates transcription factors. ${ }^{15,23} \mathrm{~A}$ line of data indicated that the expression of Notch-1 and NICD was upregulated after cerebral ischemia. ${ }^{2,14,24,25}$ Wang et al. reported that Notch-1 and NICD were predominantly expressed in doublecortinpositive (newborn neuronal) cells, but that Jagged-1 was expressed mainly in GFAP-positive cells. ${ }^{24}$ However, 2 recent publications showed that Notch-1, NICD, and Jagged-1 were expressed in astrocytes..$^{14,25}$ Recently, it was reported that Notch-1 protein levels peaked at Day 3 after ICH. ${ }^{30}$ However, our current data show a significant upregulation of Notch-1, NICD, and Jagged-1 from Day 3 to Day 14 after ICH, primarily within GFAP-positive astrocytes. These apparently contradicting results may be due to different volumes of autologous whole blood being injected into the brain and the use of different Notch-1 antibodies.

Notch-1 signaling is considered a major point of convergence between different regulators of the neuron-toastrocyte switch. ${ }^{8}$ When development proceeds, Notch-1 activation is necessary and sufficient to induce astrocyte differentiation. Expression of NICD in mouse embryonic neural stem cells results in demethylation of the GFAP promoter and, in turn, activation of GFAP expression. ${ }^{13}$ After cerebral ischemia, Notch-1 conditional knockout mice had a significantly smaller number of proliferating reactive astrocytes. ${ }^{17}$ Furthermore, a report by Kamei et al. confirmed that Jagged-1-dependent Notch signaling could mediate astrogliosis following SCI. ${ }^{7}$ In this context, we next determined whether Notch-1 signaling is involved in ICH-induced reactive astrogliosis. As expected, the increase in PCNA-positive/GFAP-positive nuclei and GFAP protein levels were attenuated after infusion of DAPT, which indicated that Notch-1 signaling contributed to re- active astrogliosis after ICH. Unfortunately, the effect of Jagged-1 on reactive astrogliosis was not addressed in this study, although a prolonged increase in Jagged-1 protein was observed, which was coincident with the expression of Notch-1.

Notch-1 has been considered a double-edged sword in cerebral ischemia. It was reported that Notch-1 signaling played a key role in normal adult and ischemia-induced neurogenesis ${ }^{24}$ and that curcumin might be protective against focal cerebral ischemia reperfusion injury as well as stimulate neurogenesis by activating the Notch signaling pathway. ${ }^{12}$ On the contrary, acute blockage of Notch-1 signaling by DAPT induces neuroprotection and neurogenesis in the neonatal rat brain after stroke. ${ }^{11}$ Notch-1 activation enhances the inflammatory response associated with focal cerebral ischemia. ${ }^{26}$ DAPT could exert a neuroprotective effect through inhibition of Notch-1 activation and

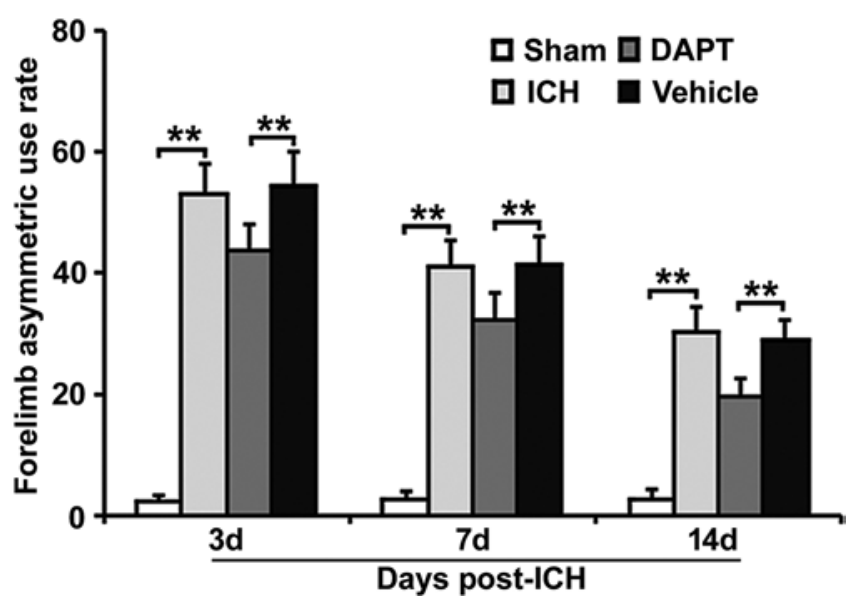

FIG. 5. Assessments and scores of behavioral tests in rats after ICH. Behavioral tests were implemented in rats after $\mathrm{ICH}$ or sham surgery. The ICH group had definitively worse impairment than the sham group at the corresponding time points. The AUR for the DAPT group was much lower than that for the vehicle group. ${ }^{* *} p<0.01 ; n=5$ rats per time point. 
neuroinflammation. ${ }^{10,25}$ Furthermore, Zou et al. reported that acupuncture could exert protective role by inhibiting Notch-1 signaling, which indicated that Notch-1 signaling might exert a detrimental effect against hemorrhagic injury. However, the authors failed to explore the mechanisms underlying a neurotoxic effect exerted by Notch-1 signaling after ICH. ${ }^{30}$ In the current study, blockage of the Notch-1 signaling pathway with DAPT improved functional outcome after ICH. Taken together, these results suggest that Notch-1 signaling might exert a detrimental effect, at least partially, through enhancing reactive astrogliosis. These apparently contradictory results may be due to differences in the time frame, animal strains, animal models of stroke, and Notch-1 signaling inhibitor used in these studies.

\section{Conclusions}

Our findings in the present study indicate that Notch-1 signaling activation is required for reactive astrogliosis after ICH. However, a number of other pathways are involved in reactive astrogliosis that remain to be further investigated. If so, identifying signaling pathways that mediate ICH-induced reactive astrogliosis may lead to new therapeutic strategies for neurological disease.

\section{Acknowledgments}

This study was supported by grants from the National Natural Science Foundation of China (Grant Nos. 30400581, 30873221, 81173175, and 81202625), the Project for New Century Excellent Talents (NCET-11-0522), the Hunan Provincial Natural Science Foundation (Grant Nos. 07JJ5007 and 10JJ2023), and the Key Laboratory of Cardiovascular and Cerebrovascular Diseases Translational Medicine (Three Gorges University, 2016KXN06).

\section{References}

1. D’Amato G, Luxán G, de la Pompa JL: Notch signalling in ventricular chamber development and cardiomyopathy. FEBS J 283:4223-4237, 2016

2. Fang M, Yuan Y, Rangarajan P, Lu J, Wu Y, Wang H, et al: Scutellarin regulates microglia-mediated TNC1 astrocytic reaction and astrogliosis in cerebral ischemia in the adult rats. BMC Neurosci 16:84, 2015

3. Gao L, Jiang T, Guo J, Liu Y, Cui G, Gu L, et al: Inhibition of autophagy contributes to ischemic postconditioning-induced neuroprotection against focal cerebral ischemia in rats. PLoS One 7:e46092, 2012

4. Givogri MI, de Planell M, Galbiati F, Superchi D, Gritti A, Vescovi A, et al: Notch signaling in astrocytes and neuroblasts of the adult subventricular zone in health and after cortical injury. Dev Neurosci 28:81-91, 2006

5. Hua Y, Schallert T, Keep RF, Wu J, Hoff JT, Xi G: Behavioral tests after intracerebral hemorrhage in the rat. Stroke 33:2478-2484, 2002

6. Jolink WM, Klijn CJ, Brouwers PJ, Kappelle LJ, Vaartjes I: Time trends in incidence, case fatality, and mortality of intracerebral hemorrhage. Neurology 85:1318-1324, 2015

7. Kamei N, Kwon SM, Ishikawa M, Ii M, Nakanishi K, Yamada K, et al: Endothelial progenitor cells promote astrogliosis following spinal cord injury through Jagged1-dependent Notch signaling. J Neurotrauma 29:1758-1769, 2012

8. Kanski R, van Strien ME, van Tijn P, Hol EM: A star is born: new insights into the mechanism of astrogenesis. Cell Mol Life Sci 71:433-447, 2014
9. LeComte MD, Shimada IS, Sherwin C, Spees JL: Notch1STAT3-ETBR signaling axis controls reactive astrocyte proliferation after brain injury. Proc Natl Acad Sci U S A 112:8726-8731, 2015

10. Li S, Zyang X, Wang Y, Ji H, Du Y, Liu H: DAPT protects brain against cerebral ischemia by down-regulating the expression of Notch 1 and nuclear factor $\kappa B$ in rats. Neurol Sci 33:1257-1264, 2012

11. Li Z, Wang J, Zhao C, Ren K, Xia Z, Yu H, et al: Acute blockage of Notch signaling by DAPT induces neuroprotection and neurogenesis in the neonatal rat brain after stroke. Transl Stroke Res 7:132-140, 2016

12. Liu S, Cao Y, Qu M, Zhang Z, Feng L, Ye Z, et al: Curcumin protects against stroke and increases levels of Notch intracellular domain. Neurol Res 38:553-559, 2016

13. Namihira M, Kohyama J, Semi K, Sanosaka T, Deneen B, Taga T, et al: Committed neuronal precursors confer astrocytic potential on residual neural precursor cells. Dev Cell 16:245-255, 2009

14. Nardai S, Dobolyi A, Pál G, Skopál J, Pintér N, Lakatos K, et al: Selegiline promotes NOTCH-JAGGED signaling in astrocytes of the peri-infarct region and improves the functional integrity of the neurovascular unit in a rat model of focal ischemia. Restor Neurol Neurosci 33:1-14, 2015

15. Palermo R, Checquolo S, Bellavia D, Talora C, Screpanti I: The molecular basis of Notch signaling regulation: a complex simplicity. Curr Mol Med 14:34-44, 2014

16. Pekny M, Wilhelmsson U, Pekna M: The dual role of astrocyte activation and reactive gliosis. Neurosci Lett 565:3038,2014

17. Shimada IS, Borders A, Aronshtam A, Spees JL: Proliferating reactive astrocytes are regulated by Notch-1 in the periinfarct area after stroke. Stroke 42:3231-3237, 2011

18. Sofroniew MV: Molecular dissection of reactive astrogliosis and glial scar formation. Trends Neurosci 32:638-647, 2009

19. Stump G, Durrer A, Klein AL, Lütolf S, Suter U, Taylor V: Notch1 and its ligands Delta-like and Jagged are expressed and active in distinct cell populations in the postnatal mouse brain. Mech Dev 114:153-159, 2002

20. Sukumari-Ramesh S, Alleyne CH Jr, Dhandapani KM: Astrocyte-specific expression of survivin after intracerebral hemorrhage in mice: a possible role in reactive gliosis? J Neurotrauma 29:2798-2804, 2012

21. Suzuki S, Tanaka K, Nogawa S, Ito D, Dembo T, Kosakai A, et al: Immunohistochemical detection of leukemia inhibitory factor after focal cerebral ischemia in rats. J Cereb Blood Flow Metab 20:661-668, 2000

22. Tsai CF, Thomas B, Sudlow CL: Epidemiology of stroke and its subtypes in Chinese vs white populations: a systematic review. Neurology 81:264-272, 2013

23. Wang H, Zang C, Liu XS, Aster JC: The role of Notch receptors in transcriptional regulation. J Cell Physiol 230:982988, 2015

24. Wang X, Mao X, Xie L, Greenberg DA, Jin K: Involvement of Notch1 signaling in neurogenesis in the subventricular zone of normal and ischemic rat brain in vivo. J Cereb Blood Flow Metab 29:1644-1654, 2009

25. Wang Z, Huang W, Zuo Z: Perioperative aspirin improves neurological outcome after focal brain ischemia possibly via inhibition of Notch 1 in rat. J Neuroinflammation 11:56, 2014

26. Wei Z, Chigurupati S, Arumugam TV, Jo DG, Li H, Chan SL: Notch activation enhances the microglia-mediated inflammatory response associated with focal cerebral ischemia. Stroke 42:2589-2594, 2011

27. Yang S, Song S, Hua Y, Nakamura T, Keep RF, Xi G: Effects of thrombin on neurogenesis after intracerebral hemorrhage. Stroke 39:2079-2084, 2008

28. Zhang Y, He K, Wang F, Li X, Liu D: Notch-1 signaling 
regulates astrocytic proliferation and activation after hypoxia exposure. Neurosci Lett 603:12-18, 2015

29. Zhou C, Yamaguchi M, Kusaka G, Schonholz C, Nanda A, Zhang JH: Caspase inhibitors prevent endothelial apoptosis and cerebral vasospasm in dog model of experimental subarachnoid hemorrhage. J Cereb Blood Flow Metab 24:419431, 2004

30. Zou W, Chen QX, Sun XW, Chi QB, Kuang HY, Yu XP, et al: Acupuncture inhibits Notch1 and Hes1 protein expression in the basal ganglia of rats with cerebral hemorrhage. Neural Regen Res 10:457-462, 2015

\section{Disclosures}

The authors report no conflict of interest concerning the materi- als or methods used in this study or the findings specified in this paper.

\section{Author Contributions}

Conception and design: HJ Zhou, Zhong. Acquisition of data: Zhong, Cui, Yang. Analysis and interpretation of data: HJ Zhou, Zhong, Tang. Drafting the article: Zhong. Critically revising the article: Tang, QM Zhang, JH Zhou, Q Zhang, Gong, ZH Zhang, Mei. Reviewed submitted version of manuscript: all authors. Approved the final version of the manuscript on behalf of all authors: HJ Zhou. Statistical analysis: Zhong. Administrative/ technical/material support: HJ Zhou. Study supervision: HJ Zhou.

\section{Correspondence}

Hua-Jun Zhou, Institute of Neurology, China Three Gorges University, 183 Yiling Rd., Yichang, Hubei 443003, China. email: zhouhuajun02@126.com. 